\title{
POINT-COUNTABILITY AND COMPACTNESS
}

\author{
H. H. WICKE AND J. M. WORRELL, JR.
}

\begin{abstract}
We prove that if a countably compact space $X$ has an open cover $\mathscr{U}=\bigcup\left\{\mathscr{V}_{n}: n<\omega\right\}$ such that each $x \in X$ is in at least one but not more than countably many elements of some $\mathfrak{V}_{n}$, then some finite subcollection of $\mathscr{U}$ covers $X$. We apply the theorem in proving several metrization theorems for countably compact spaces and discuss consequences of weak $\delta \theta$-refinability, a concept implicit in the statement of the theorem.
\end{abstract}

1. Introduction. The purpose of this article is to supply a proof of the following theorem, which was stated (for the $T_{1}$ case and in different terminology) as Theorem (iv) in [19], ${ }^{1}$ and to present some applications.

1.1. TheOrem. Suppose $X$ is a countably compact topological space and 91 is the union of a countable collection $\left\{\Upsilon_{n}: n<\omega\right\}$ of collections of open subsets of $X$ such that each $x \in X$ is in at least one element but not in more than countably many elements of some $\Upsilon_{n}$. Then some finite subcollection of $\vartheta_{1}$ covers $X$.

Theorem (iv) was stated for comparison with Theorems (i)-(iii) of [19] which concerned $\theta$-refinability, a concept introduced there. A proof of (iv) was not given since the article's major concern was with other topics.

The theorem has an intrinsic interest which is heightened by the relation it bears to some questions concerning the properties of weak $\theta$-refinability [5] and quasi-developability [4], studies of which appeared after the publication of [19]. In particular, the theorem gives an affirmative answer to the question as to whether countably compact spaces which are weakly $\theta$-refinable are compact (listed as an open problem in [11]). This result, together with Bennett and Lutzer's result that quasi-developable spaces are weakly $\theta$-refinable [5] and Bennett's theorem that compact quasi-developable Hausdorff spaces are metrizable [4], yields that all Hausdorff countably compact quasi-developable spaces are metrizable, answering a question in [4].

Implicit in the statement of Theorem 1.1 is the covering property of weak $\delta \theta$-refinability (Definition 2.1), which carries further the generalization of covering properties which imply compactness in the presence of countable compactness.

Presented to the Society, January 26, 1975; received by the editors January 28, 1975.

AMS (MOS) subject classifications (1970). Primary 54D20; Secondary 54D30, 54E20.

Key words and phrases. (Countably) compact, (weakly) $\theta$-refinable, (weakly) $\delta \theta$-refinable, $p$-space, quasi-developable space, $\theta$-base, $G_{\delta}$-diagonal, primitive base, primitive structures, diagonal a primitive set of interior condensation.

'The work on Theorem (iv) was supported by the United States Atomic Energy Commission in 1964 when the authors were associated with Sandia Laboratories. 
For these reasons, we feel that it is appropriate to publish a proof of the theorem. We are indebted to several mathematicians for requesting that we do so.

Notation and terminology. Our set-theoretic usage corresponds to that of [9]. Thus $\omega$ denotes the first infinite ordinal and $m<\omega$ means that $m$ is a positive integer. If $A$ is a set, $|A|$ denotes its cardinal number. A space $X$ is called countably compact (in accord with customary usage) if and only if every countable open covering of $X$ has a finite subcollection which covers $X$. In our proof we use the consequence of this that every infinite set $A$ has an $\omega$-accumulation point, i.e., a point $x$ such that every open set containing $x$ contains infinitely many points of $A$. If $\mathcal{V}$ is a collection of sets we define $\operatorname{order}(\mathcal{T}, x)$ by $|\{V \in \mathcal{V}: x \in V\}|$. We use $\mathcal{V}^{*}$ to denote $\cup \mathcal{V}$.

Proof of 1.1. We use the notation of 1.1. For each $n<\omega$, let $C_{n}=\{x \in$ $\left.X: 0<\operatorname{order}\left(\mathscr{V}_{n}, x\right) \leqslant \omega\right\}$. Let $C\left(\vartheta_{l}\right)$ denote the collection of all subsets of $X$ that are covered by a countable subcollection of 91 . If $X \in C(\nabla)$, the theorem will follow. If $X \notin C$ (थ) ), let $n_{0}$ denote the first $m<\omega$ such that

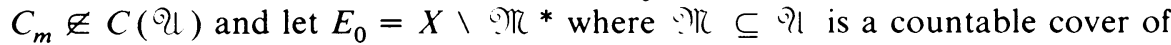
$C_{j}$ for all $j<n_{0}$. Suppose $k<\omega$ and $E_{j}$ and $n_{j}$ are such that for all $j \leqslant k$ : (1) $E_{j}$ is a closed set in $X,(2) n_{j}$ is the first $m<\omega$ such that $E_{j} \cap C_{m} \notin C$ ( 91$)$, (3) $E_{j+1} \subseteq E_{j}$ (if $E_{j+1}$ is defined), (4) $E_{j} \cap C_{m}=\varnothing$ for all $m<n_{j}$. Suppose $\widetilde{T} \subseteq$ Q1 is countable and covers $B=E_{k} \backslash \sim_{n_{k}}^{*}$. By the proof of Theorem 18 of [10, p. 8], there exists $M \subseteq\left(E_{k} \cap C_{n_{k}}\right) \backslash \mathcal{V}^{*}$ such that $\left(E_{k} \cap C_{n_{k}}\right) \backslash \mathcal{V}^{*}$ $\subseteq \cup\left\{V \in \mathcal{V}_{n_{k}}: V \cap M \neq \varnothing\right\}$ and no element of $\mathcal{V}_{n_{k}}$ contains two points of $M$. Thus, since $M \subseteq E_{k} \backslash \mathcal{V}^{*} \subseteq \mathcal{V}_{n_{k}}^{*}$ and $E_{k}$ is closed, $M$ has no $\omega$ accumulation points, so $M$ is finite. This implies that $E_{k} \cap C_{n_{k}} \in E(\mathcal{Q L})$, a contradiction. Hence $B \notin(C(\mathcal{Q}))$ and there exists $m<\omega$ such that $B \cap$ $C_{m} \notin C(\mathcal{U})$. Let $n_{k+1}$ be the first such $m$. Let $\mathcal{Q} \int \subseteq \mathcal{Q}$ be a countable cover of $B \cap C_{j}$ for all $j<n_{k+1}$. Let $\left.E_{k+1}=B \backslash 9.1\right)^{*}$. Then (1)-(4) hold for $j=k+1$. Hence sequences $\left\langle E_{k}: k\langle\omega\rangle\right.$ and $\left\langle n_{k}: k\langle\omega\rangle\right.$ exist satisfying (1)-(4) for all $j<\omega$. By (4), $\cap\left\{E_{k}: k<\omega\right\}=\varnothing$, which contradicts the countable compactness of $X$. Thus $X \in C(\vartheta l)$.

2. Applications. To state the applications we need several definitions. Let $X$ be a space and suppose $\vartheta_{0} / 5$ is a collection of subsets of $X$ of the form $\cup\left\{\Upsilon_{n}\right.$ : $n<\omega\}$. Consider these conditions on 0.10 :

(1) For each $x \in X$, there is an $n<\omega$ such that $0<\operatorname{order}\left(\Upsilon_{n}, x\right) \leqslant \omega$.

(2) For each $x \in X$, there is an $n<\omega$ such that $0<\operatorname{order}\left(\sim_{n}, x\right)<\omega$.

(3) For each $n<\omega, \mathcal{V}_{n}$ covers $X$.

2.1. Definition. Suppose $X$ is a space and every open covering of $X$ has an open refinement $थ$ satisfying (1). Then $X$ is called weakly $\delta \theta$-refinable. If, in addition to (1), थS satisfies (3), then $X$ is called $\delta \theta$-refinable [3]. If every open covering of $X$ has an open refinement ifl satisfying (2), $X$ is called weakly $\theta$-refinable [5]. If, in addition to (2), "ll satisfies (3), then $X$ is called $\theta$-refinable [19].

2.2. Definition. A collection ilf is called $\sigma$-distributively point-countable provided it is a collection of the form $\cup\left\{\widetilde{T}_{n}: n<\omega\right\}$ such that for each $x \in \cup$ Qif 1 , there is an $n<\omega$ such that $0<\operatorname{order}\left(\check{\Upsilon}_{n}, x\right) \leqslant \omega$.

The following diagram gives inclusion relationships among the covering 
properties of Definition 2.1 and other classically studied properties. An arrow from $Q$ to $\mathscr{B}$ means that the class of spaces having property $Q$, is a subclass of the class of spaces having $\mathscr{B}$. None of the arrows may be reversed.

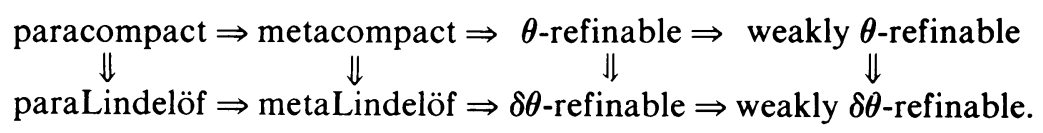

2.3. THEOREM. Every closed and countably compact subset of a weakly $\delta \theta$-refinable space is compact.

Proof. Immediate from Theorem 1.1 and the definitions involved.

2.4. COROLlARY. If a countably compact space has any of the properties listed in the diagram, then it is compact.

For the paracompact case this is due to Dieudonne [8], for metacompact spaces to Arens and Dugundji [2], for metaLindelöf spaces to Aquaro [1], for $\theta$-refinable spaces to Worrell and Wicke [19], and for $\delta \theta$-refinable spaces to Aull [3].

In the following theorems we use concepts not defined here. In the list below, the reference immediately following a concept contains a definition of the concept (the reference given is not necessarily the original source): $M$-space, $p$-space, $\beta_{b}$-space, $\beta_{c}$-space [12]; $w \Delta$-space, Burke's p-space criterion [6]; quasi-complete [7]; $\theta$-base [19]; $G_{\delta}$-diagonal, quasi-developable [4]; primitive base, primitive set of interior condensation, primitive structure of (countably) compact type [17].

2.5. THEOREM. A Hausdorff $M$-space is a paracompact $p$-space if and only if it is weakly $\delta \theta$-refinable.

Proof. Direct from the definitions and 2.3.

2.6. THEOREM. Every weakly $\delta \theta$-refinable regular $T_{0} w \Delta$-space satisfies Burke's p-space criterion.

Proof. This is a direct consequence of Theorem 1.4 of [6].

2.7. THEOREM. In a weakly $\delta \theta$-refinable regular space $X$ the following are equivalent:

(a) $X$ satisfies Burke's criterion.

(b) $X$ is a quasi-complete space.

2.8. THEOREM. If a $\beta_{c}$-space is not a $\beta_{b}$-space, then it is not locally weakly $\delta \theta$-refinable.

2.9. THEOREM. If a space having a primitive structure of countably compact type does not have a primitive structure of compact type, then it is not locally weakly $\delta \theta$-refinable.

Theorems 2.8 and 2.9 are consequences of 2.3 and the definitions involved. Theorem 2.7 may be proved analogously to Theorem 1.4 of [6].

2.10. THEOREM. Suppose $X$ is a countably compact Hausdorff space. Then the following are equivalent: 
(a) $X$ is compact metric.

(b) $X$ is quasi-developable.

(c) $X$ is weakly $\delta \theta$-refinable and has a $G_{\delta}$-diagonal.

(d) $X$ is weakly $\delta \theta$-refinable and its diagonal is locally a primitive set of interior condensation.

(e) $X$ can be covered by a $\sigma$-distributively point-countable collection of open sets each of which is weakly $\delta \theta$-refinable and has a primitive base.

(f) $X$ can be covered by a $\sigma$-distributively point-countable collection of open sets each of which is quasi-developable (equivalently, has a $\theta$-base).

Proof. That (a) $\rightarrow$ (b) $\rightarrow$ (f) is easily verified. By [ 5, Proposition 7] and [16, Theorem 2.4], (f) $\rightarrow$ (e). If (e) holds, Theorem 1.1 implies that $X$ is covered by a finite set of open weakly $\delta \theta$-refinable sets. Thus $X$ is weakly $\delta \theta$-refinable and is therefore compact. Since having a primitive base locally implies having a primitive base (this is noted in [16]; it is a consequence of a general lemma concerning open primitive sequences [15]), $X$ is compact and has a primitive base. Hence $X$ is metrizable [14], so (e) $\rightarrow$ (a). Clearly (a) $\rightarrow(c) \rightarrow(d)$. If $X$ satisfies (d), then its diagonal is a primitive set of interior condensation (see remark just above concerning [15]). By 2.3, $X$ is compact. Hence the diagonal is a set of interior condensation by the proof of Theorem 5.1 of [17] and thus $X$ is metrizable by [13]. Hence $(\mathrm{d}) \rightarrow(\mathrm{a})$, and the proof is complete.

2.11. REMARK. Weak $\delta \theta$-refinability is an abstraction of $\theta$-refinability in the presence of which countable compactness implies compactness. An abstraction of another sort which does not have this property is discussed in [20] as property $\mathrm{A}$. Every regular $\beta_{c}$-space having $\mathrm{A}$ is quasi-complete and every regular $\beta_{b}$-space having $A$ is a $p$-space in the sense of [6]. Thus with Theorem 2.8 we have that regular weakly $\delta \theta$-refinable $\beta_{c}$-spaces having $\mathrm{A}$ are $p$-spaces in the sense of [6]. On the other hand, there are countably compact $T_{1}$ collectionwise normal $p$-spaces having $A$ which are not weakly $\delta \theta$-refinable [20], there are Tychonoff weakly $\theta$-refinable $\beta_{b}$-spaces which are not $p$-spaces [18], and the space of countable ordinals with the order topology is a countably compact $p$-space which does not have $A$ and is not weakly $\delta \theta$-refinable. Thus neither abstraction solves the problem of characterizing nontrivially those Tychonoff $\beta_{b}$-spaces which are $p$-spaces. In the case of essentially $T_{1}$ spaces having bases of countable order property $\mathrm{A}$ is equivalent to $\theta$-refinability.

\section{REFERENCES}

1. G. Aquaro, Point countable coverings in countably compact spaces, General Topology and its Relations to Modern Analysis and Algebra, II (Prague, 1967), pp. 39-41.

2. R. Arens and J. Dugundji, Remark on the concept of compactness, Portugaliae Math. 9(1950), 141-143. MR12, 434.

3. C. E. Aull, A generalization of a theorem of Aquaro, Bull. Austral. Math. Soc. 9(1973), $105-108$.

4. H. R. Bennett, On quasi-developable spaces, General Topology and Appl. 1(1971), no. 3, 253-262. MR 44 \#5921.

5. H. R. Bennett and D. J. Lutzer, $A$ note on weak $\theta$-refinability, General Topology and Appl. 2(1972), 49-54. MR46 \#853.

6. D. K. Burke, On p-spaces and ws-spaces, Pacific J. Math. 35(1970), 285-296. MR43 \#3986.

7. G. G. Creede, Concerning semi-stratifiable spaces, Pacific J. Math. 32(1970), 47-54. MR40 \#8006. 
8. J. Dieudonné, Une généralisation des espaces compacts, J. Math. Pures Appl. (9) 23(1944), 65-76. MR7, 134.

9. J. D. Monk, Introduction to set theory, McGraw-Hill, New York, 1969. MR44 \# 3877.

10. R. L. Moore, Foundations of point set theory, rev. ed., Amer. Math. Soc. Colloq. Publ., vol. 13, Amer. Math. Soc., Providence, R.I., 1962. MR27 \#709.

11. M. E. Rudin, Lecture notes, Univ. of Wyoming Topology Conf. (Laramie, Wy., 1974).

12. H. H. Wicke, Open continuous images of certain kinds of $M$-spaces and completeness of mappings and spaces, General Topology and Appl. 1(1971), no. 1, 85-100. MR43 \#8060.

13. On spaces whose diagonal is a set of interior condensation, Notices Amer. Math. Soc. 19(1972), A-657. Abstract \#696-54-16.

14. H. H. Wicke and J. M. Worrell, Jr., Characterizations of absolute sets of interior condensation, Notices Amer. Math. Soc. 20(1973), A-401. Abstract \# 704-G6.

15. _ Topological completeness of first countable Hausdorff spaces. II, Fund. Math. (to appear).

16. _ A characterization of primitive bases, Proc. Amer. Math. Soc. 50 (1975), 443-450.

17. _ Primitive structures in general topology (Proc. 1974 Topology Conf., Univ. of North Carolina at Charlotte), Studies in topology, edited by N. M. Stavrakas and K. R. Allen, Academic Press, New York, 1975, pp. 581-599.

18. J. M. Worrell, Jr., A perfect mapping not preserving the p-space property, presented at Pittsburgh Conf. on General Topology, 1970.

19. J. M. Worrell, Jr. and H. H. Wicke, Characterizations of developable topological spaces, Canad. J. Math. 17(1965), 820-830. MR32 \#427.

20 . , Remarks on a property of $\theta$-refinable spaces, Notices Amer. Math. Soc. 21(1974), A-622. Abstract \#717-G16.

Department of Mathematics, Ohio University, Athens, Ohio 45701 